\title{
Pigment-Dispersing Factor and GABA Synchronize Cells of the Isolated Circadian Clock of the Cockroach Leucophaea maderae
}

\author{
Nils-Lasse Schneider and Monika Stengl \\ Department of Biology, Animal Physiology, Philipps University of Marburg, D-35032 Marburg, Germany
}

\begin{abstract}
Pigment-dispersing factor-immunoreactive circadian pacemaker cells, which arborize in the accessory medulla, control circadian locomotor activity rhythms in Drosophila as well as in the cockroach Leucophaea maderae via unknown mechanisms. Here, we show that circadian pacemaker candidates of the accessory medulla of the cockroach produce regular interspike intervals. Therefore, the membrane potential of the cells oscillates with ultradian periods. Most or all oscillating cells within the accessory medulla are coupled via synaptic and nonsynaptic mechanisms, forming different assemblies. The cells within an assembly share the same ultradian period (interspike interval) and the same phase (timing of spikes), whereas cells between assemblies differ in phase. Apparently, the majority of these assemblies are formed by inhibitory GABAergic synaptic interactions. Application of pigment-dispersing factor phase locked and thereby synchronized different assemblies. The data suggest that pigment-dispersing factor inhibits GABAergic interneurons, resulting in disinhibition and phase locking of their postsynaptic cells, which previously belonged to different assemblies. Our data suggest that phase control of action potential oscillations in the ultradian range is a main task of the circadian pacemaker network. We hypothesize that neuropeptide-dependent phase control is used to gate circadian outputs to locomotor control centers.
\end{abstract}

Key words: pigment-dispersing factor; circadian pacemakers; ultradian oscillations; circadian clock; GABA; synchronization; resonance

\section{Introduction}

The presence of an endogenous circadian clock in the brain of an animal was demonstrated first in the cockroach Leucophaea maderae (Nishiitsutsuji-Uwo and Pittendrigh, 1968; Sokolove, 1975; Page, 1982). However, the cellular basis of the clock remained elusive until pigment-dispersing factor (PDF)-immunoreactive (IR) neurons were proposed as circadian pacemaker candidates in the fruitfly and the cockroach (Homberg et al., 1991, 2003; Helfrich-Förster and Homberg, 1993; Stengl and Homberg, 1994; Helfrich-Förster, 1995). In Drosophila, these neurons express clock genes essential for circadian rhythmicity such as period and timeless (Helfrich-Förster, 1995; Kaneko and Hall, 2000) (for review, see Helfrich-Förster, 2004). The PDF-IR neurons are closely associated with the accessory medulla (AMe), a small neuropil in the optic lobe (Reischig and Stengl, 1996; Sato et al., 2002; Bloch et al., 2003; Sehadová et al., 2003; Závodská et al., 2003), which was identified as the circadian clock of the cockroach (Reischig and Stengl, 2003a). The PDF-IR cells are essential ele-

\section{Received July 15, 2004; revised Feb. 10, 2005; accepted April 4, 2005}

We thank Drs. J. C. Hall (Brandeis University, Waltham, MA) and U. Homberg (Department of Biology, University of Marburg) for helpful revisions of this manuscript and Drs. H. Dircksen and D. Nässel (University of Stockholm, Stockholm, Sweden) for generously supplying PDF peptides. We are thankful for considerable improvement of this manuscript by unknown referees. In addition, we thank Drs. A. Pikovsky and M. Rosenblum (Department of Physics, University of Potsdam, Potsdam, Germany) for helpful discussions of oscillator theory and synchronization of oscillators.

Correspondence should be addressed to Monika Stengl, Department of Biology, Animal Physiology, Philipps University of Marburg, Karl von Frisch Strasse, D-35032 Marburg, Germany. E-mail: stengl@staff.uni-marburg.de. DOI:10.1523/JNEUROSCI.5138-A-04.2005

Copyright $\odot 2005$ Society for Neuroscience $\quad$ 0270-6474/05/255138-10\$15.00/0 ments of the circadian clock controlling locomotor activity rhythms (Stengl and Homberg, 1994; Reischig and Stengl, 2003a). Associated with the AMe are $\sim 250$ cells. More than $60 \%$ of these express nuclear PERIOD immunoreactivity (Fischer, 2002).

In the cockroach AMe, as in the mammalian suprachiasmatic nucleus (SCN), GABA-IR processes are abundant (Van den Pol and Tsujimoto, 1985; Moore and Speth, 1993; Petri et al., 1995, 2002). In addition, many different neuropeptide-IR neurons form clock subcompartments, which are dense knots of arborizations (noduli) with looser internodular neuropil and a shell of tracts embracing the AMe (Petri et al., 1995; Reischig and Stengl, 1996, 2003b). Approximately 25 GABA-IR neurons and the GABA-IR distal tract, the presumptive light entrainment pathway from the compound eye, arborize extensively in the noduli of the AMe (Petri et al., 2002; Reischig and Stengl, 2003b). The PDF-IR neurons, which branch in the internodular neuropil, control locomotor rhythms via projections to locomotor control centers in the superior lateral protocerebrum (Stengl and Homberg, 1994; Reischig and Stengl, 2003a). In addition, three of the 12 PDF-IR neurons connect both accessory medullas (AMae) and apparently serve to synchronize both pacemakers (Reischig and Stengl, 2004). Consistent with this hypothesis, PDF acts as a nonphotic input signal into the clock, delaying circadian locomotor activity rhythms (Petri and Stengl, 1997). In Drosophila, PDF is also assumed to be a circadian coupling signal that synchronizes clock cells in the midbrain to control locomotor activity rhythms (Peng et al., 2003; Lin et al., 2004). 
Although considerable information about the molecular machinery of the core circadian pacemaker is available (Honma and Honma, 2003), little is known about the physiological properties of the clock. It is mostly unresolved how circadian coupling is accomplished and how the neuropeptides act within the clock. Here, we tested in extracellular recordings from excised AMae of the cockroach L. maderae whether GABA and PDF affect AMe neurons via modulation of neuronal activity. We discovered that AMe neurons express ultradian action potential oscillations (period-interspike interval) and are grouped into phase-locked assemblies via GABA-dependent synaptic interactions. We hypothesize that PDF-dependent phase locking of assemblies gates clock output to locomotor control centers.

\section{Materials and Methods}

All experiments were performed on AMae of adult male cockroaches during the day. Breeding colonies of the cockroach (L. maderae) were kept at the University of Marburg at $30^{\circ} \mathrm{C}$ and $30 \%$ humidity in $12 \mathrm{~h}$ light/dark cycles, with lights on from 7:00 A.M. to 7:00 P.M. Animals were provided with dry dog food, potatoes, and water ad libitum.

The experimental animals were decapitated, and the head capsule was opened to expose the optic lobes. After removal of fat body and the perineurial sheath around the optic lobes, the AMe was excised with a glass pipette (diameter, $150 \mu \mathrm{m}$; Flaming/Brown Micropipette Puller, model P-97; Sutter Instruments, Novato, CA). The location of the AMe was easily discernible beneath the bifurcation of a characteristic trachea. All experiments were performed at constant light. Altogether, 32 AMae were used in experiments involving application of GABA and PDF (see below)

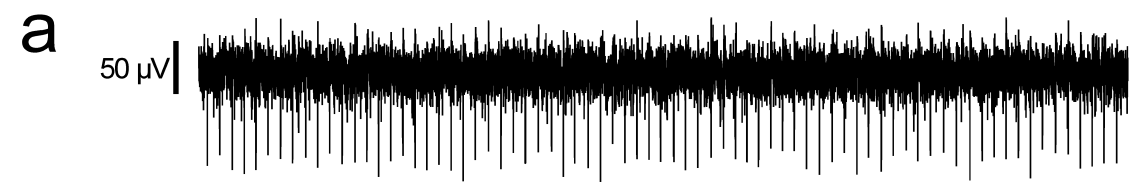

b

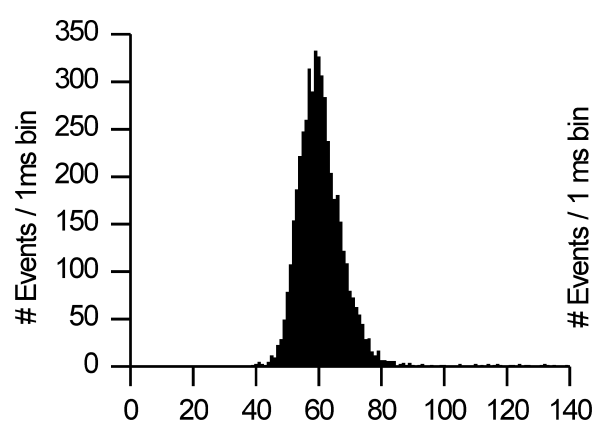

d

Interevent-interval / ms

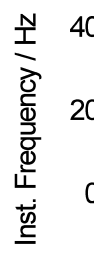

7
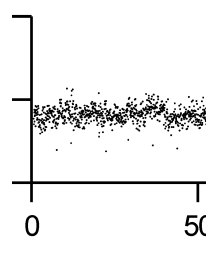

50

$$
100
$$
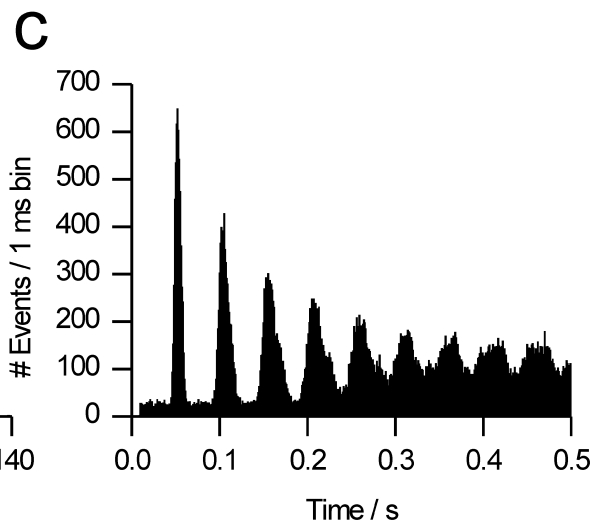

Figure 1. $\boldsymbol{a}-\boldsymbol{d}$, Neurons of the AMe fire very regularly. $\boldsymbol{a}$, Extracellular recording from an excised AMe with baseline noise of $\sim 45 \mu \mathrm{V}$. The summed action potential activity (events) appears to originate from more than one cell, because it comprises different event amplitudes that occur as downward deflections of the baseline. $\boldsymbol{b}$, The interevent-interval distribution peaks sharply at $\sim 60 \mathrm{~ms}$, indicating that all recorded cells fire synchronously with the same phase and the same interevent interval. $\boldsymbol{c}$. Multiple defined peaks in the autocorrelogram show the high regularity of spiking activity of AMe neurons. $\boldsymbol{d}$, The instantaneous frequency (Inst. Frequency) (1/interevent interval) plot reveals a defined band of $\sim 16.7 \mathrm{~Hz}$. or of pharmacological agents; additional recordings involved controls (application of vehicle only) and changes of the ionic conditions. In summary, 623 experiments in 32 separate recordings were performed.

For the extracellular recordings, the AMe was transferred to a Petri dish (diameter, $4 \mathrm{~cm}$ ). All chemicals used were purchased from SigmaAldrich (Taufkirchen, Germany). The osmolarity of the extracellular saline ( $156 \mathrm{~mm} \mathrm{NaCl}, 4 \mathrm{~mm} \mathrm{KCl}, 6 \mathrm{~mm} \mathrm{CaCl}$, 10 mm HEPES, 5 mm glucose, $0.01 \mathrm{~g} / \mathrm{L}$ phenol red, $\mathrm{pH}$ 7.1) was adjusted with mannitol to $380 \mathrm{mOsm}$ In the $\mathrm{Ca}^{2+}$-free extracellular solution, $\mathrm{CaCl}_{2}$ was replaced by $1 \mathrm{~mm}$ EGTA. PDF (NSELINSLLSLPKNMNDA-NH ${ }_{2}$ ), GABA, picrotoxin (PTX), and tetrodotoxin (TTX) were dissolved and diluted in saline. Bicuculline was dissolved in chloroform and diluted in saline. The tissue was continuously superfused with $10 \mathrm{ml}$ of saline per hour at room temperature. Drugs were applied to the tissue either by pressure ejection via glass capillaries (Picospritzer II; General Valve Corporation, Fairfield, $\mathrm{NJ}$ ) or via bath application to a chamber with a volume of $5 \mathrm{ml}$ and a flow rate of $30-40 \mathrm{ml} / \mathrm{h}$. The doses of applied materials varied according to the following two delivery modes: (1) pressure ejection (PDF, 24-800 fmol; GABA, 1-5 pmol; PTX, 100-200 pmol; bicuculline, 0.4-2 nmol), and (2) bath application (GABA, ranging from 1 to $1000 \mu \mathrm{M}$; PTX, ranging from 1 to $1000 \mu \mathrm{M}$; bicuculline, $10 \mu \mathrm{M}$; TTX, $0.1 \mu \mathrm{M}$ ).

Extracellular electrical activity of the excised AMe was recorded with glass electrodes $(0.3-1.5 \mathrm{M} \Omega$ ) connected to an extracellular amplifier (EXT-01C/DPA 2F; NPI Electronics, Tamm, Germany). Because of the low resistance of the recording electrodes, multiunit action potentials (events) were recorded as upward and/or downward deflections of the baseline. The output of the amplifier was high-pass filtered $(3 \mathrm{~Hz})$ to eliminate electrode offset and low-pass filtered $(1.5 \mathrm{kHz})$ to avoid highfrequency noise and aliasing. The signal was digitized (DIGIDATA 1322A; Molecular Devices, Union City, CA) with a sampling rate of 5 $\mathrm{kHz}$ and stored on a disk for additional analysis. Event detection via threshold search was performed off-line with SPIKE II software (Cambridge Electronic Design, Cambridge, UK).

The mean frequency (number of events per second) was calculated to evaluate the effects of applied drugs on the firing rate of the cells. Interevent-interval distributions were generated using $1 \mathrm{~ms}$ bin width and different periods (depending on the experiment, with a minimum of $100 \mathrm{~s}$ ) at different time points of the experiment to identify changes in the regularity of the electrical activity. Instantaneous frequency (1/interevent interval) plots were calculated over the whole time course of the experiments to visualize the regularity of electrical activity.

Electrical activity originating from irregularly spiking neurons or from neurons spiking with different rates or phases that are not integer multiples of each other result in a broad cloud of instantaneous frequencies. A defined narrow band of instantaneous frequencies indicates that all recorded cells fire action potentials very regularly at the same or integer multiples of the same interevent interval (harmonic frequencies). Parallel bands in the instantaneous frequency plot indicate that at least two cells fire with the same or integer multiples of the same interevent interval but with different phase relationships. If more than one cell fires with different noninteger multiples of interevent intervals and with different phase, the bands cross each other (Pikovsky et al., 2001). Fusion of parallel bands to one band indicates that the recorded cells now fire with the same phase and the same ultradian period (same interevent intervals). Autocorrelograms were generated using a bin width of $1 \mathrm{~ms}$ for an interval of $1 \mathrm{~s}$ over 
5-30 min periods to identify rhythmic firing patterns (Groves et al., 1978). Regular interevent intervals cause multiple peaks in the autocorrelogram (Tepper et al., 1995).

\section{Results}

In extracellular recordings, we tested whether the neuropeptide PDF and the neurotransmitter GABA affect the electrical activity of interneurons of the AMe of the cockroach L. maderae. We show that neurons of the AMe are ultradian oscillators that are grouped into phase-locked assemblies via GABAergic synaptic interactions. PDF transiently phase locked assemblies of neurons apparently via inhibition of GABAergic interneurons, thereby disinhibiting their postsynaptic cells. We hypothesize that this disinhibition activates PDF-IR outputs to locomotor control centers via resonance (see Fig. 9 ). We assume that circadian clocks are indispensable for phase control of neuronal oscillators also at the time scale of milliseconds, which underlies temporal encoding of the brain.

Extracellular recordings of electrical events of the excised AMe lasted for several hours (Fig. 1). The average peak-to-peak noise amplitude was $\sim 40 \mu \mathrm{V}$. Event amplitudes ranged from 50 to $150 \mu \mathrm{V}$ and were observed as upward and downward deflections from the baseline, depending on the impedance ratio between the recorded neurons, the recording electrode, and the indifferent electrode. Application of the sodium channel blocker TTX $\left(10^{-8}\right.$ M) reversibly blocked all electrical events within several hours after application to the bath solution (data not shown; $n=3$ experiments in three different preparations).

Extracellular recordings usually were multiunit recordings and were composed of activity from more than one cell with different event amplitudes (Fig. 1a). Less than five recordings appeared to result from single cells. These showed constant action potential amplitude and regular firing pattern, which resulted in a sharp peak of the interevent-interval histogram (data not shown for single cells) (Fig. $1 b$, multiunit recording). Likewise, $>80 \%$ of the multiunit recordings (of 32 preparations) showed sharp multiple peaks in the autocorrelograms, indicating regular firing modes of recorded neurons (Tepper et al., 1995) (Figs. $1 c, 2 b, d, f)$.

In $72 \%$ of all recordings, spontaneous activity occurred in one to seven parallel bands in the instantaneous frequency plot (1/ interevent interval; see Materials and Methods). Activity in one band originates from spontaneously active cells that fire at the same phase and the same or integer multiples of the same interspike interval (harmonic frequencies) (Figs. 1d, 2a). Parallel bands indicate that neurons with the same or with integer multiples of the same frequency fire with a stable phase difference (Figs. 2c, 3c) (see Figs. 5c, $7 c, 8 c$ ). Thus, spontaneously active ultradian oscillators are grouped into assemblies. All cells within $\mathrm{b}$
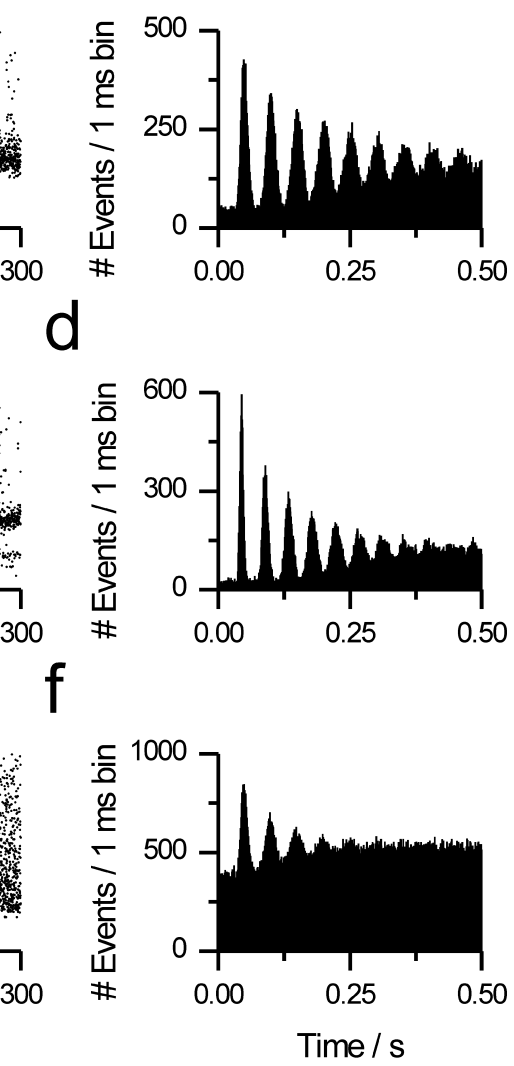

Time/s

\section{Time / s}

Figure 2. $\boldsymbol{a}-\boldsymbol{f}$, Instantaneous frequency (Inst. Frequency) plots reveal that AMe neurons fire regularly at the same or at different phases. $\boldsymbol{a}$, One narrow band in the instantaneous frequency plot indicates that cells fire with the same phase and the same or integer multiples of the same interevent interval. $\boldsymbol{b}$, The regular interevent intervals result in multiple peaks in the (the same or integer multiples of the same interevent interval but with different phase show oscillatory spiking activity. $\boldsymbol{e}$, If cells spike with the same or integer multiples of the same interevent interval but with many different phase relationships, the instantaneous frequency plots do not show regularity. $\boldsymbol{f}$, Autocorrelation analysis, however, still reveals regularity in the spiking pattern.

an assembly fire phase locked with the same or with harmonic frequencies, whereas cells between assemblies fire at a stable phase difference.

To examine whether the regular action potential activity of AMe neurons is a property of the single cells or whether it is caused by synaptic synchronization of irregularly spiking neurons, we superfused the AMe with $\mathrm{Ca}^{2+}$-free or high $\mathrm{Mg}^{2+}$ saline to inhibit neurotransmitter release (Fig. 3). Application of $\mathrm{Ca}^{2+}$ free saline resulted in an increase of the mean event frequency and amplitude, apparently as a result of release from synaptic inhibition. In 19 of 23 experiments (involving 13 preparations), the sharp band of instantaneous frequencies broadened and gradually increased to a higher frequency level. Simultaneously, several new bands of lower frequency appeared parallel to the highfrequency band. Thus, the previously single assembly split into different assemblies. Superfusion with saline containing $10 \mathrm{~mm}$ $\mathrm{Mg}^{+2}$ mimicked the effects of low-divalent solutions $(n=3)$ (data not shown), suggesting that the observed changes in activity are caused by loss of synaptic connections and not only by changes in the extracellular $\mathrm{Ca}^{2+}$ concentration.

Several events appear to underlie these observed phenomena. Neurons of one assembly, firing in synchrony with the same phase, appear to gradually shift to a new constant phase while 

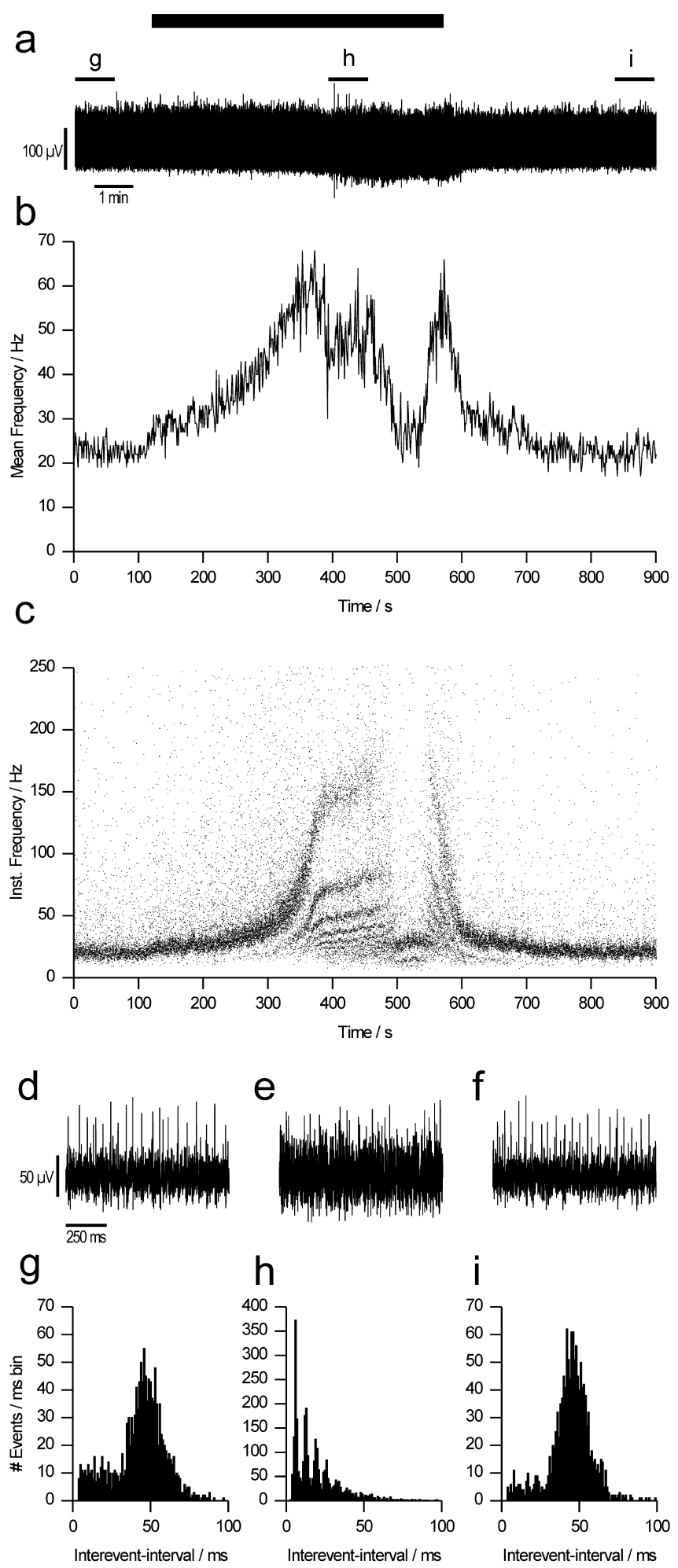

Figure 3. $\boldsymbol{a}-\boldsymbol{i}$, Regular spiking AMe neurons are synchronized to the same phase and frequency by inhibitory synaptic interactions. $\boldsymbol{a}$, The black bar indicates the substitution of extracellular saline for $\mathrm{Ca}^{2+}$-free solution to disrupt synaptic activity in the extracellular recording of the excised AMe. The same time axis is used in $\boldsymbol{a}-\boldsymbol{c}$. Thin bars $(g, h, i)$ indicate the time window for the interevent-interval distributions in $\boldsymbol{g}-\boldsymbol{i}$. The $\mathrm{Ca}^{2+}$-free solution causes a larger variability of event amplitudes and a rise in the mean frequency $(\boldsymbol{b})$, apparently resulting from disinhibition and shift of phase as seen in c. The instantaneous frequency (Inst. Frequency) plot (c) and the interevent-interval distributions $(\boldsymbol{g}-\boldsymbol{i})$ illustrate that, in normal saline, all cells fire in synchrony at the same phase and a lower action potential frequency. After disruption of synaptic activity, the sharp $22.7 \mathrm{~Hz}$ band continuously increases to a higher instantaneous frequency while new parallel bands appear. Thus, without synaptic contacts, more cells become active, but increasing their firing frequency. In addition, previously silent neurons appear to discharge action potentials but at the same interspike interval and with constant phase relationship to the firing neurons. Thus, without synaptic communication, cells remained coupled ultradian oscillators but split into different assemblies that fired with constant phase difference and with higher frequency. After returning to extracellular saline with $6 \mathrm{mM} \mathrm{Ca}^{2+}$ concentrations, the parallel bands smoothly declined and fused to one lower frequency band $(n=10)$. These findings indicate that all cells gradually returned to firing in synchrony. Apparently, via inhibitory synaptic interactions again, they formed an assembly and fired at the same phase and at the same lower frequency (Fig. 3c). Frequently, a sharp drop in instantaneous frequency occurred when we switched back to high-Ca ${ }^{2+}$. containing extracellular saline but before complete exchange of the low-divalent extracellular solution. It is possible that this sudden inhibition of activity is caused by sudden synchronous release of an inhibitory neurotransmitter either via $\mathrm{Ca}^{2+}$ release from intracellular stores or via $\mathrm{Ca}^{2+}$ influx.

Dense GABA immunostaining in the AMe (Petri et al., 2002) suggests that GABA essentially is responsible for the inhibitory synaptic interactions between AMe neurons. Application of GABA (1-5 pmol via picospritzer; $1 \mu \mathrm{M}$ to $1 \mathrm{mM}$ via bath) resulted in a dose-dependent strong inhibition of electrical activity (Fig. 4$)$ in $98 \%$ of all experiments ( $n=453 ; 15$ preparations). Reduced spiking activity developed within 1 to $2 \mathrm{~s}$ after application. Activity returned within 10 to $100 \mathrm{~s}$, depending on the applied dose. In approximately one-third of all experiments in instantaneous frequency plots, GABA-dependent phase locking of cells was observed before electrical activity ceased (data not shown). Application of the vertebrate $\mathrm{GABA}_{\mathrm{A}}$ receptor antagonist bicuculline $(0.4-2 \mathrm{nmol})$ showed no effect $(n=5$; five preparations). Because bicuculline is also without effect on $\mathrm{GABA}_{\mathrm{A}}$ receptors in other invertebrate preparations, whereas the chloride channel blocker picrotoxin was used successfully as a GABA channel antagonist (Lee et al., 2003; Sattelle et al., 2003), we used picrotoxin. Picrotoxin ( $1 \mu \mathrm{M}$ to $1 \mathrm{~mm}$ ) reproducibly opposed GABA-dependent inhibition. Picrotoxin application increased electrical activity, the instantaneous frequency band smoothly increased, and several parallel bands appeared. Thus, in the presence of picrotoxin, cells split into different assemblies and remained synchronized but resumed a stable phase difference and fired with higher frequencies ( $n=6$; five preparations) (Fig. 5). The density of the bands increased after picrotoxin application and smoothly shifted to higher instantaneous frequencies. This indicates that previously silent cells gradually started to fire. However, these neurons always remained phase locked at a stable phase difference, because several parallel bands appeared, not just noise, or only one band at a higher instantaneous frequency. Because picrotoxin is not specific for GABA receptors but blocks different chloride channels, we cannot exclude that it might also inhibit other ligand-gated chloride channels such as glutamate receptors.

Application of PDF (24-800 fmol with picospritzer) during the day (Zeitgebertime ZT 4-11) changed the electrical activity of AMe neurons in $78 \%$ of all experiments. Peptide application ei-

$\leftarrow$

all cells maintain coupling and fire very regularly with the same or integer multiples of the same interevent interval but with a constant phase difference $(\boldsymbol{h})$. The sudden, reversible inhibition at $500 \mathrm{~s}$ in coccurred spontaneously, possibly resulting from store-dependent $\mathrm{Ca}^{2+}$ release. $\boldsymbol{d}-\boldsymbol{f}$, Original trace at an expanded time scale (at time points $\mathrm{g}$, $\mathrm{h}$, and $\mathrm{i}$ in $\boldsymbol{a}$ ). 
ther transiently decreased $(n=58$; seven preparations) or increased ( $n=76$; four preparations) neuronal activity after delays of 1-100 s (Fig. 6). In low-divalent solutions, which disrupt synaptic transmission, PDF always led to neuronal inhibition ( $n=5$, involving three preparations) (Table 1), suggesting that the excitatory effects in normal saline were indirect effects through inhibitory interneurons. Indeed, after PDF-induced transient activations, removal of extracellular $\mathrm{Ca}^{2+}$ or elevation of $\mathrm{Mg}^{2+}$ abolished the PDF effect ( $n=7$; three preparations). In the presence of picrotoxin (Table 1), in two experiments, PDF-dependent activation was seen ( $n=2$; one preparation) whereas in 11 experiments, PDF-dependent inhibition was observed ( $n=11$; one preparation). Thus, PDF-dependent inhibition/ disinhibition appears not to be mediated via chloride channel opening. PDFdependent increases in the event amplitudes (Figs. 6a, 7a) were observed, which resulted from either disinhibition of previously inhibited cells or PDF-dependent synchronization of cells, which were not phase locked previously to the same phase. High-amplitude events were abruptly and transiently elicited superimposed on the background activity accompanied by an increase in the mean frequency (Fig. 6a); thus, PDF disinhibited new units. In other recordings, the event amplitudes increased gradually while the mean frequency decreased (Figs. 7a,b). These PDF-dependent increases in the event amplitudes resulted from synchronization of units, which previously belonged to different assemblies, as shown in instantaneous frequency plots (Fig. 7c).

In recordings with several instantaneous frequency bands, PDF caused a transient, smooth fusion of the bands to a higher synchronized instantaneous frequency compared with the lowest frequency band before application but to a lower instantaneous frequency compared with the highest band before application (Fig. 7). Because the previously parallel bands are fused to one dense band, PDF strictly synchronizes and phase locks the recorded units to the same phase (Fig. $7 c$ ). Because after PDF application the fused band maintains a higher instantaneous frequency than the lowest frequency band before application, PDF disinhibited AMe neurons ( $n=16$; five preparations) (Fig. 7 ). At the same time, PDF inhibited previously active cells, because the fused band maintains a lower synchronized instantaneous frequency compared with the highest frequency band before application.

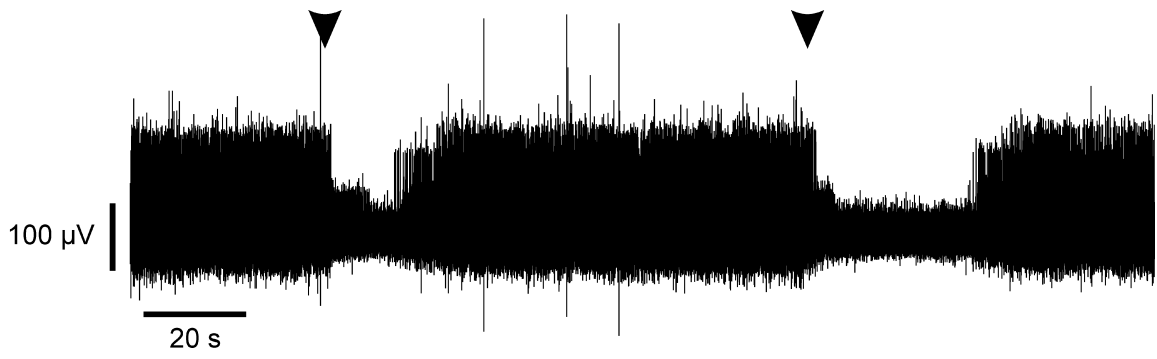

Figure 4. The neurotransmitter GABA inhibits AMe neurons dose dependently. Application of $0.1 \mathrm{pmol}$ of GABA (left arrowhead) and 0.5 pmol of GABA (right arrowhead) inhibits electrical activity of the AMe neurons with different durations.
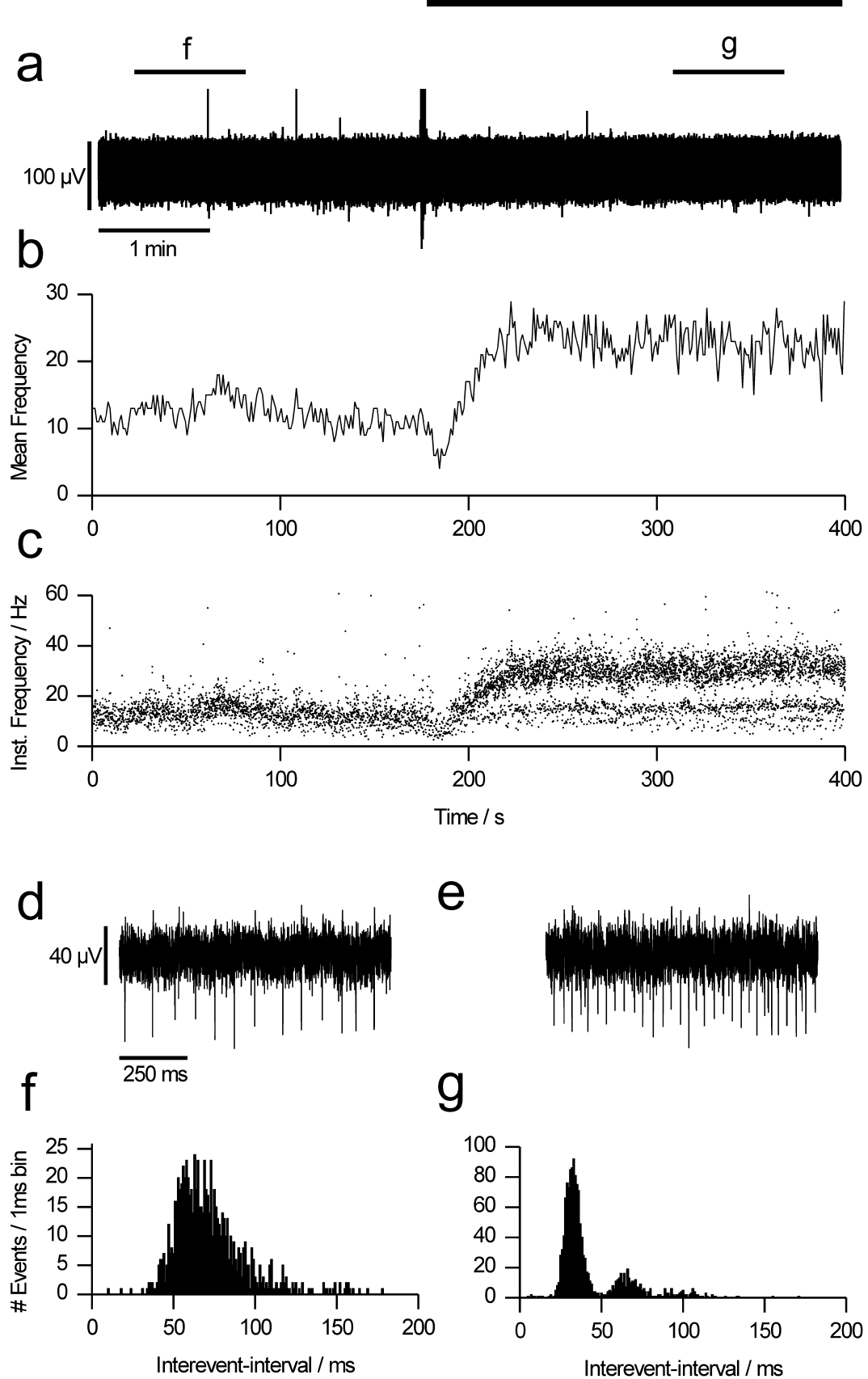

Figure 5. In the presence of the $\mathrm{Cl}^{-}$channel antagonist picrotoxin (which also blocks $\mathrm{GABA}_{\mathrm{A}}$ receptors), AMe neurons remain synchronized but fire with a stable phase difference and a higher frequency. $\boldsymbol{a}$, Original recording trace over $400 \mathrm{~s}$. The black bar marks bath application of 1 mм picrotoxin. Thin bars $(f, g)$ indicate the time frames of interevent-interval distributions in $\boldsymbol{f}$ and $\boldsymbol{g}$. c, The instantaneous frequency (Inst. Frequency) plot shows phase desynchronization of AMe neurons during picrotoxin application. $\boldsymbol{d}, \boldsymbol{e}$, Original trace at an expanded time scale (at time points fand $\mathrm{g}$ in $\boldsymbol{a}$ ). $\boldsymbol{f}, \boldsymbol{g}$, The interevent-interval distributions show that, before and during picrotoxin application, the AMe neurons maintain regular firing activity. 


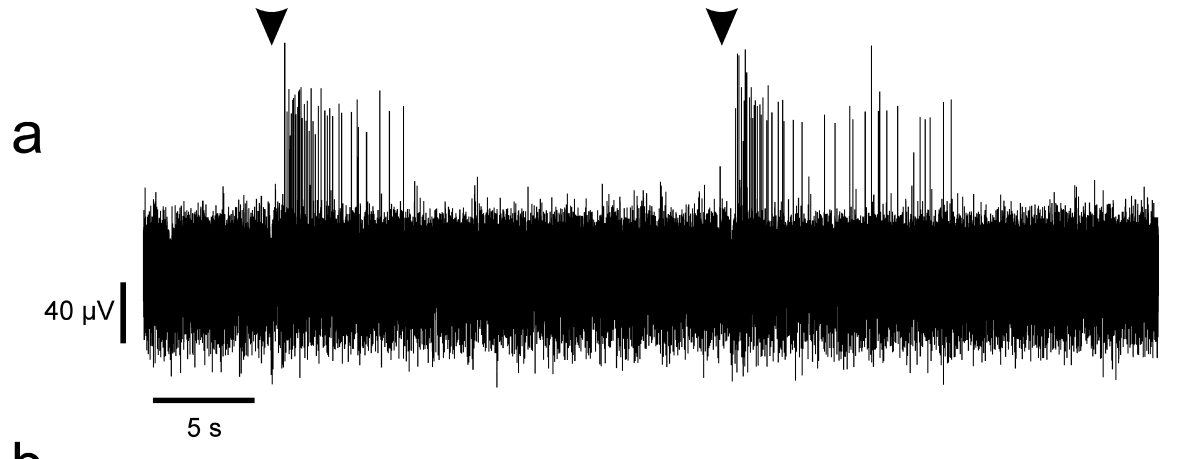

b

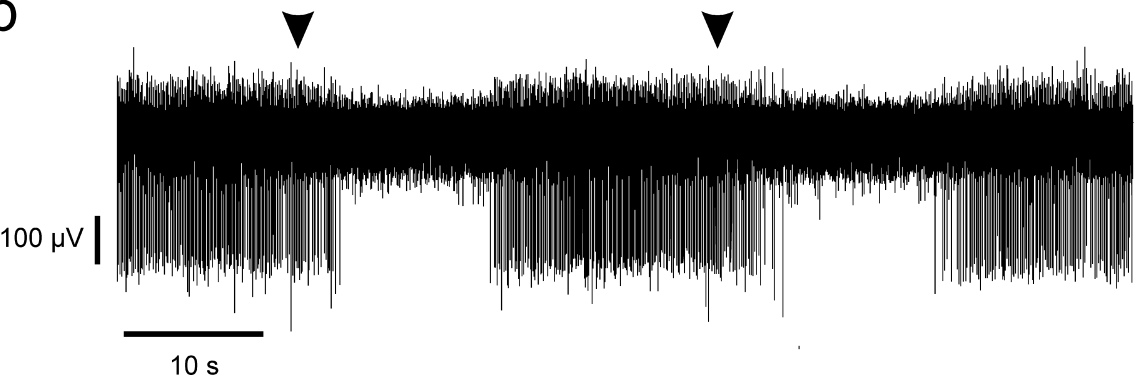

Figure 6. PDF leads to activation or inhibition of AMe neurons. $\boldsymbol{a}$, After PDF application of $75 \mathrm{fmol}$ (arrowheads), large events appeared superimposed on the background activity $(\boldsymbol{b})$. In another recording, $75 \mathrm{fmol}$ PDF inhibited AMe neurons, repetitively.

Table 1. Effects of PDF application to excised accessory medullas of the cockroach $L$. maderae in different extracellular salines

\begin{tabular}{llll}
\hline Saline & $\begin{array}{l}\mathrm{Ca}^{2+} \text {-free } \\
\text { saline }\end{array}$ & $\begin{array}{l}\text { Saline plus } \\
\text { picrotoxin }\end{array}$ & \\
\hline Activation & No effect & Not tested & $n=7$ (3 preparations) \\
Activation & Not tested & Activation & $n=2$ (1 preparation) \\
Inhibition & Inhibition & Not tested & $n=5$ (3 preparations) \\
Inhibition & Not tested & Inhibition & $n=11$ (1 preparation) \\
\hline
\end{tabular}

During the fusion of the bands, the event amplitudes in the original recordings transiently increased (by $\sim 20 \%$ ) (Fig. $7 a$ ) while the mean frequency dropped. This effect of PDF application indicates superposition of action potentials of different cells, which now fire at the same phase and the same or integer multiples of the same interevent intervals. In addition, the mean frequency increased while the peptide-dependent fusion of the instantaneous frequency bands gradually disappeared. This particular result suggests that during washout (because of perfusion of the dish) of PDF, the phases of the neurons smoothly drifted apart again until their previous stable frequency and phase relationships were restored. The smoothness of transitions from parallel bands to one fused band and back to parallel bands indicates that the recorded AMe neurons always remained synchronized. Thus, PDF only synchronized the phase of perpetually coupled AMe neurons, which previously belonged to different assemblies. Control applications of vehicle resulted in none of the physiological modulations as described for the PDF effects (Fig. 8) $(n=9$; five preparations).

\section{Discussion}

Extracellular recordings from the excised AMe, the circadian clock of the cockroach $L$. maderae, investigated the function of PDF and of GABA on neuronal spike discharges. Circadian pacemaker candidates fire action potentials with regular interspike intervals and are coupled via synaptic and nonsynaptic mechanisms. They are organized into phase-locked assemblies via GABAergic interneurons. Different assemblies distinguished by a stable phase difference were transiently synchronized to the same phase and period by PDF. We assume that PDF-dependent phase locking activates outputs to locomotor control centers (Fig. 9).

\section{AMe neurons are synchronized by GABA}

Picrotoxin application caused a pronounced increase of the instantaneous frequency, together with the appearance of new parallel instantaneous frequency bands. This suggests that $\mathrm{Cl}^{-}$channeldependent inhibitory synaptic transmission synchronizes the phase of AMe neurons. Because GABA immunoreactivity is prominent in the AMe and because, in most of our recordings, GABA inhibited neuronal activity, the majority of inhibitory connections appear to be mediated via $\mathrm{GABA}_{\mathrm{A}}$ receptors. Because picrotoxin is not specific for $\mathrm{GABA}_{\mathrm{A}}$ receptors, we cannot exclude that other inhibitory neurotransmitters are also involved.

The occurrence of several parallel bands in instantaneous frequency plots indicates that different phase-locked assemblies of AMe neurons fire at a constant phase difference. Because picrotoxin further split and multiplied parallel bands while GABA fused them, GABAergic interneurons appear to form different neuronal assemblies within the AMe, keeping cells within an assembly synchronized and phase locked to the same phase (below or above spiking threshold). A similar self-organization into phase-locked assemblies with stable phase differences was demonstrated in computer simulations for coupled oscillators (Klevecz at al., 1984, 1991; Kunz and Achermann, 2003).

In the mammalian SCN, GABA is the predominant neurotransmitter (Moore and Speth, 1993; Wagner et al., 1997; De Jeu and Pennartz, 2002) and has been shown to synchronize circadian pacemaker neurons via $\mathrm{GABA}_{\mathrm{A}}$ receptor-dependent inhibitions (Strecker et al., 1997; Liu and Reppert, 2000; Shinohara et al., 2000; Michel and Colwell, 2001). Synchronization of neurons via GABAergic interneurons is also used in other parts of the insect brain such as the antennal lobe, in which neuronal synchronization appears to be important for odor coding (Christensen et al., 2000; Laurent, 2002).

AMe neurons show sharp parallel bands in the instantaneous frequency plots even after disruption of synaptic connections, which indicates that they are still synchronized but now maintain a stable phase difference. Therefore, there must be additional, nonsynaptic interactions between the AMe neurons such as gap junctions, glia-neuron interactions, or nonsynaptic release of neuromediators (Shinohara et al., 1995; Jiang et al., 1997; Tamada et al., 1998; Michel and Colwell, 2001; Cheng et al., 2002; Schaap et al., 2003). We are currently testing with dye injections as well as pharmacologically whether gap junctions couple AMe neurons. Whether the multitude of different peptides that occur in the circadian clock of $L$. maderae might contribute to nonsynaptic synchronization remains to be examined (Petri et al., 1995). 
AMe neurons are synchronized by PDF In L. maderae, the 12 PDF-IR neurons of the AMe apparently serve in different circuits of the circadian clock (Reischig and Stengl, 2002, 2003a,b, 2004). The small, weakly staining PDF-IR neurons appear to be local neurons of the AMe; two large and one medium PDF-IR neurons directly connect both AMae, whereas the other large and medium cells form outputs to various midbrain and optic lobe targets (Reischig and Stengl, 2004). Thus, in L. maderae, as in Drosophila, PDF-IR neurons are circadian pacemaker candidates and, at the same time, serve as nonphotic clock inputs and outputs. Their peptide PDF is assumed to synchronize the bilaterally symmetric clocks, the pacemaker cells within one AMe, and apparently postsynaptic pacemakers in the midbrain (Helfrich-Förster et al., 1998; Homberg et al., 2003; Lin et al., 2004).

Drosophila mutants without PDF lose circadian locomotor activity rhythms in constant darkness (Renn et al., 1999), whereas ectopic expression of PDF causes highly increased irregular activity. This indicates that PDF regulates locomotor activity rhythms in Drosophila (HelfrichFörster et al., 2000). In the cockroach, lesion and transplantation experiments showed that PDF-IR neurons with projections to the superior medium protocerebrum are necessary and sufficient for the control of locomotor activity rhythms (Stengl and Homberg, 1994; Reischig and Stengl, 2003a). In addition, PDF injections into the vicinity of the AMe delayed the onset of locomotor activity rhythms during the late day, indicating that PDF action in the AMe affects clock outputs (Petri and Stengl, 1997). Furthermore, in Drosophila, PDF-dependent communication among clusters of neurons in the dorsal brain that contain clock-gene products seems to be necessary for maintaining the phase and amplitude of clock gene expression rhythms and for sustaining circadian activity rhythms (Peng et al., 2003; Lin et al., 2004). Thus, in the fruitfly as well as in the cockroach, PDF is assumed to be an output signal of the clock to midbrain oscillators controlling onset of locomotor activity rhythms.

Here, we show that PDF synchronized cells to the same phase with smooth phase shifts while decreasing and/or increasing their ultradian periods (interspike intervals). Apparently, PDF acts as an inhibitory peptide, because in the absence of synaptic connections, PDF only inhibited but never activated neurons. Therefore, the observed PDF-dependent activations most likely result from inhibition of inhibitory interneurons. Because PDF-dependent disinhibitions were observed in the presence of picrotoxin, in addition to GABAergic interneu-
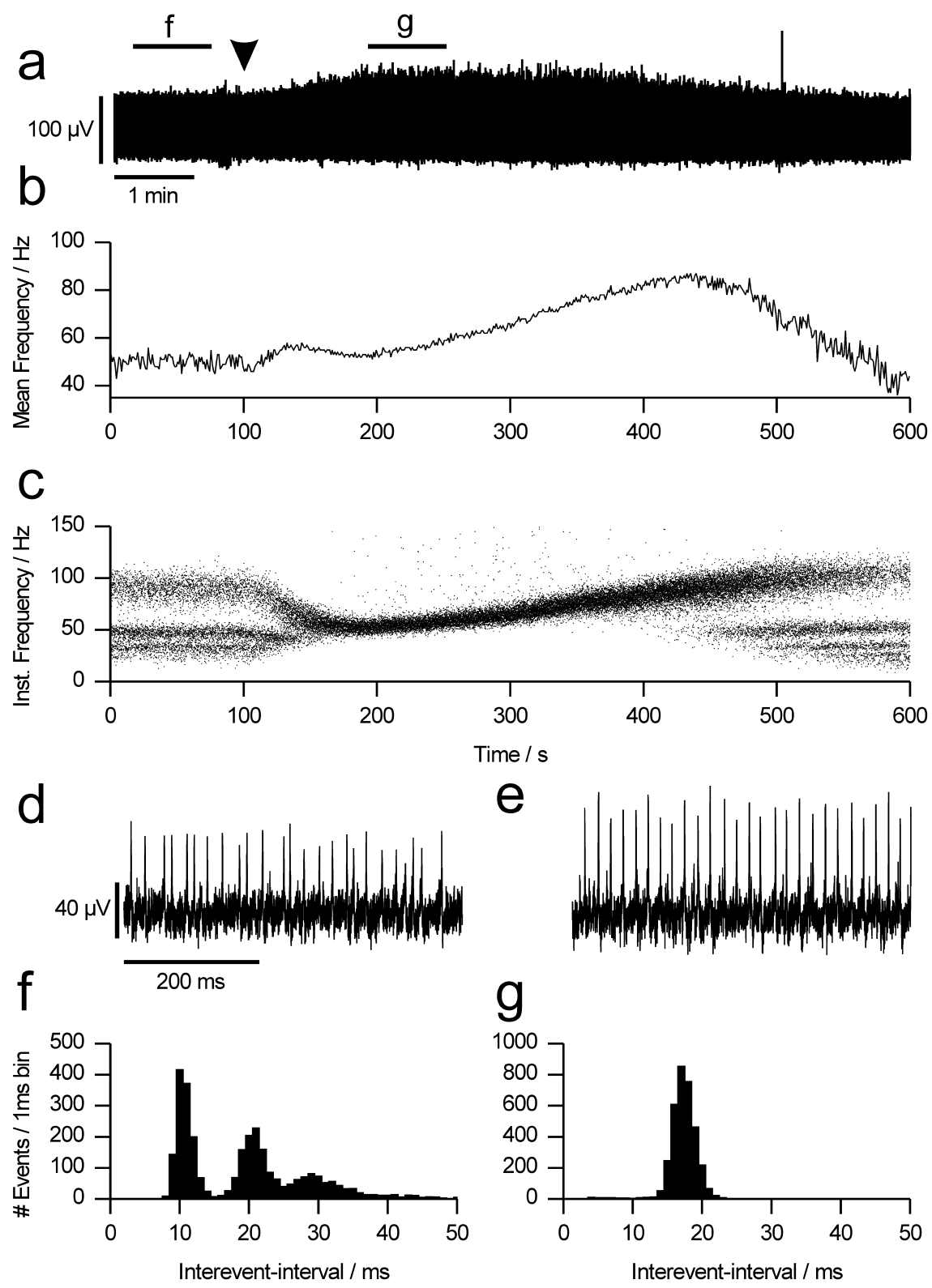

Figure 7. PDF synchronizes AMe neurons. $\boldsymbol{a}$, After application of $75 \mathrm{fmol}$ PDF (arrowhead), the event amplitudes increased continuously and transiently. Bars $(f, g)$ indicate where interevent-interval distributions were calculated. $\boldsymbol{b}$, After PDF application, there is a small transient increase in the mean frequency, followed by a transient decrease to a level that is higher than the starting level. Then, a continuous stronger rise occurs before the mean frequency declines again to a level below the starting level. Comparison with c shows that rises in the mean frequency correlate with desynchronization, whereas the declines correlate with synchronization of spiking neurons. c, The parallel, distinct bands in the instantaneous frequency (Inst. Frequency) plot indicate that at least two AMe neurons fire synchronized with the same (or at integer multiples of the same) interevent interval but at a different phase. Application of PDF synchronizes and phase locks the neurons transiently; thus, now all cells fire at the same time with the same frequency as seen in the superposition of amplitudes (compare $\boldsymbol{d}, \boldsymbol{e}$ ) and the single peak in the interevent interval distribution $(\boldsymbol{g})$. The interevent-interval distribution $(\boldsymbol{f})$ shows that at least two regularly firing synchronized cells (or assemblies of cells) with a constant phase difference are hidden in the apparently irregularly occurring events of the original recording (d). In $\boldsymbol{e}$ and $\boldsymbol{g}$, the cells are now phase locked to the same phase, and their event amplitudes superimpose.

rons, other inhibitory interneurons appear to have PDF receptors. Thus, our data suggest that synchronization of circadian clock cells within the AMe, between both AMae, and between the clock and postsynaptic oscillators is accomplished by phase locking action potential oscillations. PDF-dependent disinhibition might also gate the coupling pathway to the contralateral pacemaker center, which synchronizes both AMae. In addition, it is possible that PDF-dependent phase locking of clock cells with 

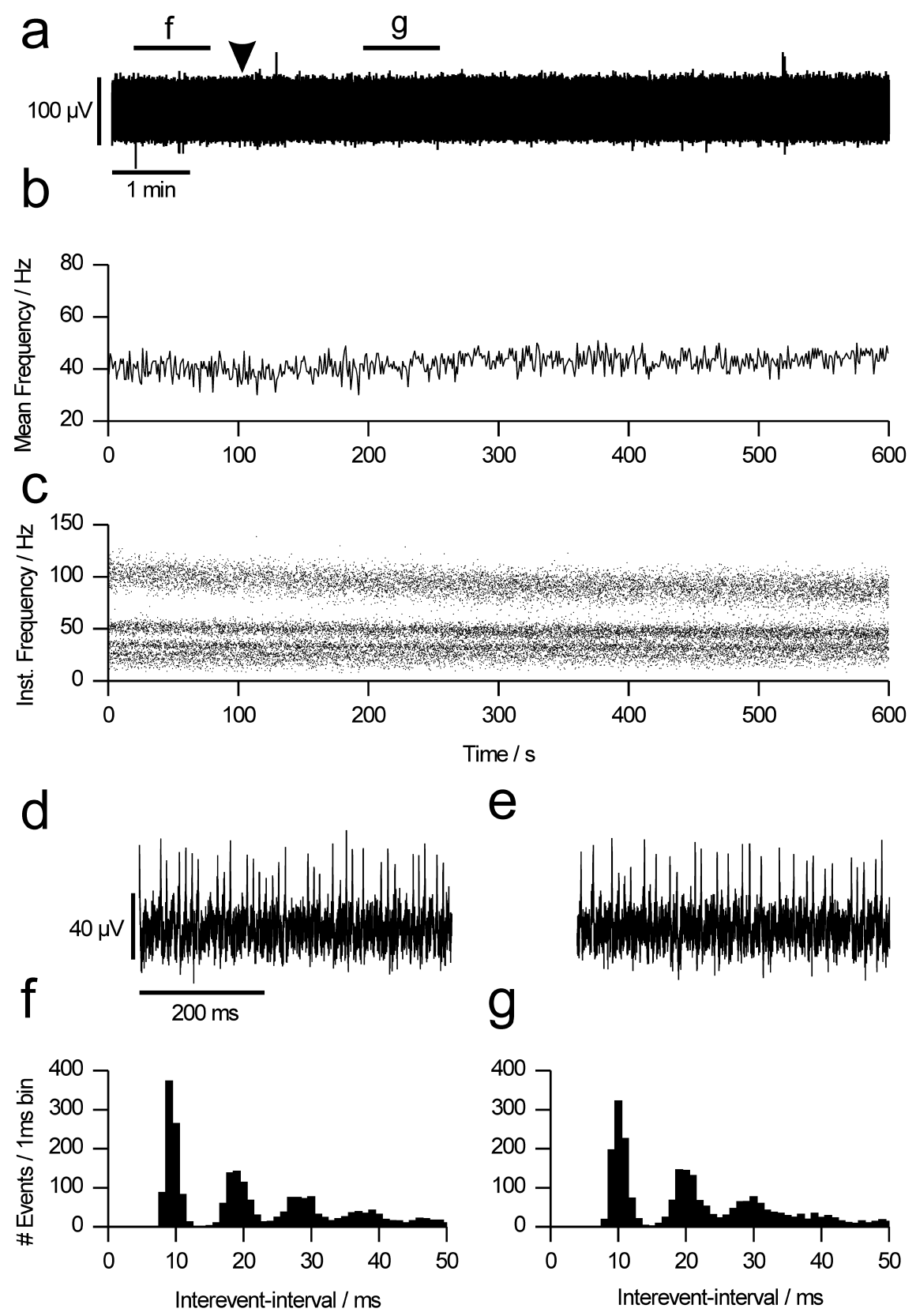

Figure 8. Control applications of vehicle do not affect AMe neurons. No vehicle-dependent changes are seen in the original recording $(\boldsymbol{a})$, the mean frequency plot $(\boldsymbol{b})$, the instantaneous frequency (Inst. Frequency) plot $(\boldsymbol{c})$, or the interevent-interval distributions $(\boldsymbol{f}, \boldsymbol{g}) . \boldsymbol{d}, \boldsymbol{e}$, Original trace at an expanded time scale (at time points $\mathrm{f}$ and $\mathrm{g}$ in $\boldsymbol{a}$ ).

PDF-IR output pathways via the anterior optic commissure to the superior medium protocerebrum (Reischig and Stengl, 2003a) might gate the onset of circadian locomotor activity during late day.

AMe neurons generate ultradian action potential oscillations Our experiments show for the first time that the large majority of neurons of the AMe fire very regularly. Because this regular spiking activity is maintained in the absence of synaptic interactions, it is likely that the discharges are generated endogenously. It is possible that the membrane potential of circadian pacemaker candidates oscillates endogenously and thereby elicits action potentials in regular interspike intervals. Regular oscillations in the membrane potential causing circadian changes in action potential frequencies have also been described in mollusc circadian pacemaker cells (Jacklet, 1988; Block et al., 1995), but it was not analyzed whether all of these also fire regularly on an ultradian scale. Whether all regularly discharging cells of the cockroach circadian clock are also endogenous circadian pacemaker neurons and vice versa remains to be examined. PDF-IR neurons apparently generate very regular interspike intervals, because intracellular recordings from neurons with the same branching pattern as PDF-IR neurons showed spontaneous discharge rates with regularly spaced spikes (Loesel and Homberg, 2001). In addition, regularly bursting neurons with arborizations in the circadian clock were recorded (Loesel and Homberg, 1998, 2001). The importance of the membrane potential for circadian oscillations is also suggested by Nitabach et al. (2002), who showed that constant hyperpolarization of the membrane of circadian pacemaker neurons in Drosophila interrupts circadian oscillations in clock gene expression in the nucleus. In the nucleus, clock gene products such as PERIOD and TIMELESS suppress their own transcription via a negative-feedback loop. Several interlocked feedback loops of different clock genes generate $24 \mathrm{~h}$ rhythms in their mRNA and protein levels (for review, see Honma and Honma, 2003). Thus, it is likely that the observed ultradian action potential rhythms and the molecular feedback loop in the nucleus are intimately connected. In the SCN of mammals, circadian pacemaker candidates with regular interevent intervals have also been observed, but the relevance of these ultradian oscillations for circadian rhythms remained unresolved (Gross and Hendriks, 1979; Shibata et al., 1984; Cahill and Menaker, 1989; Pennartz et al., 1998; Schaap et al., 1999).

Because the majority of AMe neurons fired regularly, our experiments indicate that ultradian membrane oscillations might be an important property of circadian pacemaker neurons, possibly leading to circadian scale oscillations via high-frequency oscillator coupling (Klevecz et al., 1984, 1991). Because information processing in the brain happens at the time course of milliseconds, circadian oscillators, which precisely effect timing of different physiological processes at different phases, should also be precise at the time course of milliseconds and seconds and not only at the time course of hours.

Information processing between phase-locked ultradian oscillators as presynaptic and postsynaptic cells is precise, fast, and happens with improved signal-to-noise ratio as the cells resonate, a mechanism of temporal encoding widely used in the mammalian brain (Izhikevich et al., 2003). Resonance occurs if the 

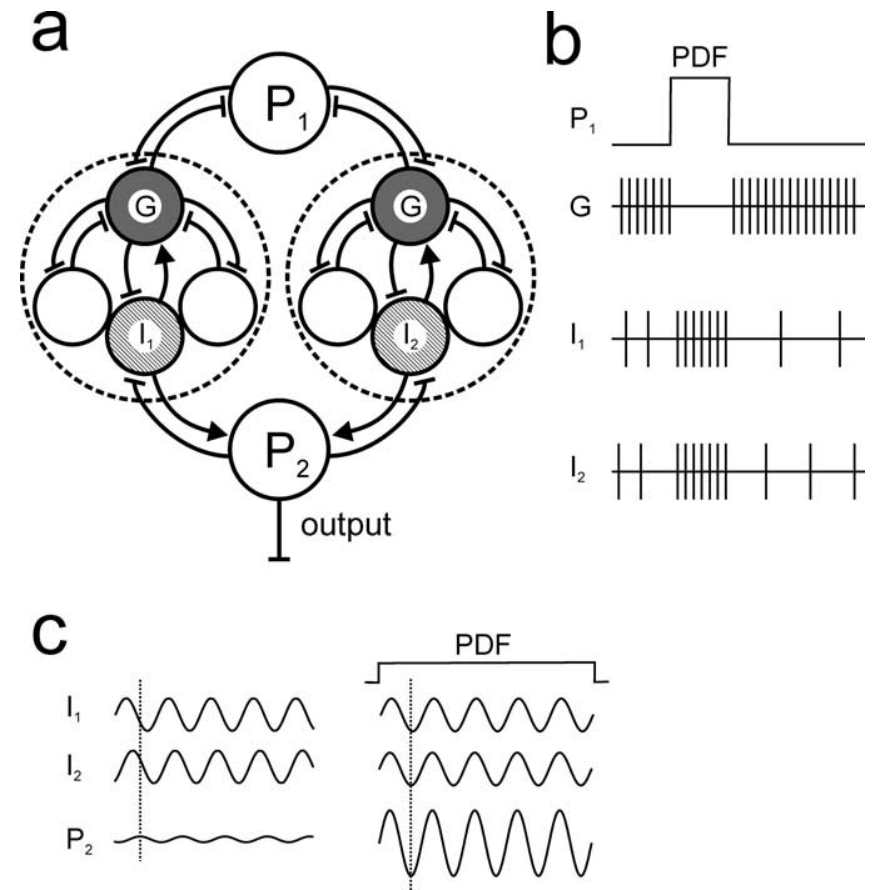

Figure 9. $\boldsymbol{a}-\boldsymbol{c}$, Model of the PDF-dependent gating mechanism. $\boldsymbol{a}$, In the AMe, GABAergic interneurons $(G)$ form assemblies of phase-locked endogenous oscillators. Within an assembly, all cells have the same phase and period, whereas between assemblies, constant phase differences are maintained via nonsynaptic, unknown mechanisms. As further illustrated with schematically drawn action potentials $(\boldsymbol{b})$, PDF release from coupling elements $\left(\mathrm{P}_{1}\right)$ phase locks assemblies via inhibition of GABAergic interneurons. Thereby, postsynaptic cells $\left(I_{1,2}\right)$ are disinhibited. The disinhibition and phase locking of different assemblies allows activation of their jointly controlled postsynaptic cells $\left(P_{2}\right)$ via resonance. $c$, When membrane potential oscillations of the presynaptic cells $\left(I_{1,2}\right)$ become phase locked with the potential oscillations of the postsynaptic cell $\left(\mathrm{P}_{2}\right)$, the postsynaptic cell resonates; the superposition of endogenous rhythmic depolarizations with input-dependent depolarizations causes $P_{2}$ to reach threshold and fire action potentials. Therefore, we hypothesize that neuropeptide-dependent phase locking of neuronal oscillators activates the output of the pacemaker centers to locomotor control centers via resonance.

postsynaptic cell expresses membrane potential oscillations in synchrony with presynaptic oscillating neurotransmitter release, and thus neurotransmitter-induced depolarizations add up with subthreshold membrane depolarizations, causing the otherwise inactive postsynaptic cell to fire (Izhikevich et al., 2003). Thus, our results suggest that the circadian clock is a network of interconnected oscillators, which uses the control of phase relationships between ultradian membrane potential oscillations to gate various outputs via neuropeptides (Fig. 9).

\section{References}

Bloch G, Solomon SM, Robinson GE, Fahrbach SE (2003) Patterns of PERIOD and pigment-dispersing hormone immunoreactivity in the brain of the Eur honeybee Apis mellifera: age- and time-related plasticity. J Comp Neurol 464:269-284.

Block G, Geusz M, Khalsa S, Michel S, Whitmore D (1995) Cellular analysis of a molluscan retinal biological clock. Ciba Found Symp 183:51-60.

Cahill GM, Menaker M (1989) Responses of the suprachiasmatic nucleus to retinohypothalamic tract volleys in a slice preparation of the mouse hypothalamus. Brain Res 479:65-75.

Cheng MY, Bullock CM, Li C, Lee AG, Bermak JC, Belluzzi J, Weaver DR, Leslie FM, Zhou QY (2002) Prokineticin 2 transmits the behavioural circadian rhythm of the suprachiasmatic nucleus. Nature 417:405-410.

Christensen TA, Pawlowski VM, Lei H, Hildebrand JG (2000) Multi-unit recordings reveal context-dependent modulation of synchrony in odorspecific neural ensembles. Nat Neurosci 3:927-931.

De Jeu M, Pennartz C (2002) Circadian modulation of GABA function in the rat suprachiasmatic nucleus: excitatory effects during the night phase. J Neurophysiol 87:834-844.

Fischer J (2002) Immunocytochemie des Uhrproteins PERIOD bei der Schabe Leucophaea maderae. Diplome thesis, Philipps University of Marburg.

Gross GA, Hendriks J (1979) Regularly firing neurones in the rat suprachiasmatic nucleus. Experientia 35:1597-1598.

Groves PM, Wilson CJ, MacGregor RJ (1978) Neuronal interactions in the substantia nigra revealed by statistical analysis of spike trains. In: Interaction between putative neurotransmitters (Garattini S, Pujol F, Samanin R, eds), pp 191-215. New York: Raven.

Helfrich-Förster C (1995) The period clock gene is expressed in central nervous system neurons which also produce a neuropeptide that reveals the projections of circadian pacemaker cells within the brain of Drosophila melanogaster. Proc Natl Acad Sci USA 92:612-616.

Helfrich-Förster C (2004) The circadian clock in the brain: a structural and functional comparison between mammals and insects. J Comp Physiol [A] 190:601-613.

Helfrich-Förster C, Homberg U (1993) Pigment-dispersing hormone-immunoreactive neurons in the nervous system of wild-type Drosophila melanogaster and of several mutants with altered circadian rhythmicity. J Comp Neurol 337:177-190.

Helfrich-Förster C, Stengl M, Homberg U (1998) Organization of the circadian system in insects. Chronobiol Int 15:567-594.

Helfrich-Förster C, Täuber M, Park JH, Mühlig-Versen M, Schneuwly S, Hofbauer A (2000) Ectopic expression of the neuropeptide pigmentdispersing factor alters behavioral rhythms in Drosophila melanogaster. J Neurosci 20:3339-3353.

Homberg U, Würden S, Dircksen H, Rao KR (1991) Comparative anatomy of pigment-dispersing hormone-immunoreactive neurons in the brain of orthopteroid insects. Cell Tissue Res 266:343-357.

Homberg U, Reischig T, Stengl M (2003) Neural organization of the circadian system of the cockroach Leucophaea maderae. Chronobiol Int 20:577-591.

Honma S, Honma K (2003) The biological clock: $\mathrm{Ca}^{2+}$ links the pendulum to the hands. Trends Neurosci 26:650-653.

Izhikevich E, Desai NS, Walcott EC, Hoppensteadt FC (2003) Bursts as a unit of neural information: selective communication via resonance. Trends Neurosci 26:161-167.

Jacklet JW (1988) Circadian pacemaker neurons: membranes and molecules. J Physiol (Paris) 83:164-171.

Jiang Z, Yang YQ, Allen CN (1997) Tracer and electrical coupling of rat suprachiasmatic nucleus neurons. Neuroscience 77:1059-1066.

Kaneko M, Hall JC (2000) Neuroanatomy of cells expressing clock genes in Drosophila: transgenic manipulations of the period and timeless genes to mark the perikarya of circadian pacemaker neurons and their projections. J Comp Neurol 422:66-94.

Klevecz RR, Kauffman SA, Shymko RM (1984) Cellular clocks and oscillators. Int Rev Cytol 184:97-128.

Klevecz RR, Pilliod J, Bolen J (1991) Autogenous formation of spiral waves by coupled chaotic attractors. Chronobiol Int 8:6-13.

Kunz H, Achermann P (2003) Simulation of circadian rhythm generation in the suprachiasmatic nucleus with locally coupled self-sustained oscillators. J Theor Biol 224:63-78.

Laurent G (2002) Olfactory network dynamics and the coding of multidimensional signals. Nat Rev Neurosci 3:884-895.

Lee D, Sue H, O'Dowd DK (2003) GABA receptors containing Rdl subunits mediate fast inhibitory synaptic transmission in Drosophila neurons. J Neurosci 23:4625-4634.

Lin Y, Stormo D, Taghert PH (2004) The neuropeptide pigment-dispersing factor coordinates pacemaker interactions in the Drosophila circadian system. J Neurosci 24:7951-7957.

Liu C, Reppert SM (2000) GABA synchronizes clock cells within the suprachiasmatic circadian clock. Neuron 25:123-128.

Loesel R, Homberg U (1998) Sustained oscillations in an insect visual system. Naturwissenschaften 85:238-240.

Loesel R, Homberg U (2001) Anatomy and physiology of neurons with processes in the accessory medulla of the cockroach Leucophaea maderae. J Comp Neurol 439:193-207.

Michel S, Colwell CS (2001) Cellular communication and coupling within the suprachiasmatic nucleus. Chronobiol Int 18:579-600. 
Moore R, Speth JC (1993) GABA is the principle neurotransmitter of the circadian system. Neurosci Lett 150:112-116.

Nishiitsutsuji-Uwo J, Pittendrigh CS (1968) Central nervous system control of circadian rhythmicity in the cockroach. II. The optic lobes, locus of the driving oscillator? Z Vgl Physiol 58:14-46.

Nitabach MN, Blau J, Holmes TC (2002) Electrical silencing of Drosophila pacemaker neurons stops the free-running circadian clock. Cell 109:485-495.

Page TL (1982) Transplantation of the cockroach circadian pacemaker. Science 216:73-75.

Peng Y, Stoleru D, Levine JD, Hall JC, Rosbash M (2003) Drosophila free-running rhythms require intercellular communication. PLoS Biol 1:E13.

Pennartz CM, De Jeu MT, Geurtsen AM, Sluiter AA, Hermes ML (1998) Electrophysiological and morphological heterogeneity of neurons in slices of rat suprachiasmatic nucleus. J Physiol (Lond) 506:775-793.

Petri B, Stengl M (1997) Pigment-dispersing hormone shifts the phase of the circadian pacemaker of the cockroach Leucophaea maderae. J Neurosci 17:4087-4093.

Petri B, Stengl M, Würden S, Homberg U (1995) Immunocytochemical characterization of the accessory medulla in the cockroach Leucophaea maderae. Cell Tissue Res 282:3-19.

Petri B, Homberg U, Loesel R, Stengl M (2002) Evidence for a role of GABA and Mas-allatotropin in photic entrainment of the circadian clock of the cockroach Leucophaea maderae. J Exp Biol 205:1459-1469.

Pikovsky A, Rosenblum M, Kurths J (2001) Synchronization. A universal concept in nonlinear sciences. In: Cambridge nonlinear science, series 12 (Chirikov B, Cvitanovic P, Moss F, Swinney H, eds), pp 27-45. Cambridge: Cambridge UP.

Reischig T, Stengl M (1996) Morphology and pigment-dispersing hormone immunocytochemistry of the accessory medulla, the presumptive circadian pacemaker of the cockroach Leucophaea maderae: a light and electronmicroscopic study. Cell Tissue Res 285:306-319.

Reischig T, Stengl M (2002) Optic lobe commissures in a three-dimensional brain model of the cockroach Leucophaea maderae: a search for the circadian coupling pathways. J Comp Neurol 443:388-400.

Reischig T, Stengl M (2003a) Ectopic transplantation of the accessory medulla restores circadian locomotor rhythms in arrhythmic cockroaches (Leucophaea maderae). J Exp Biol 206:1877-1886.

Reischig T, Stengl M (2003b) Ultrastructure of pigment-dispersing hormoneimmunoreactive neurons in a three-dimensional model of the accessory medulla of the cockroach Leucophaea maderae. Cell Tissue Res 314:421-435.

Reischig T, Stengl M (2004) Pigment-dispersing hormone (PDH)immunoreactive neurons form a direct coupling pathway between the bilaterally symmetric circadian pacemakers of the cockroach Leucophaea maderae. Cell Tissue Res 318:553-564.

Renn SC, Park JH, Rosbash M, Hall JC, Taghert PH (1999) A pdf neuropeptide gene mutation and ablation of PDF neurons each cause severe abnormalities of behavioral circadian rhythms in Drosophila. Cell 99:791-802.

Sato S, Chuman Y, Matsushima A, Tominaga Y, Shimohigashi Y, Shimohi- gashi M (2002) A circadian neuropeptide, pigment-dispersing factor$\mathrm{PDF}$, in the last-summer cicada Meimuna opalifera: cDNA cloning and immunocytochemistry. Zool Sci 19:821-828.

Sattelle DB, Bai D, Chen HH, Skeer JM, Buckinham SD, Rauh JJ (2003) Bicuculline-insensitive GABA-gated $\mathrm{Cl}^{-}$channels in the larval nervous system of the moth Manduca sexta. Invert Neurosci 3:37-43.

Schaap J, Bos NP, de Jeu MT, Geurtsen AM, Meijer JG, Pennartz CM (1999) Neurons of the rat suprachiasmatic nucleus show a circadian rhythm in membrane properties that is lost during prolonged whole-cell recording. Brain Res 815:154-166.

Schaap J, Pennartz CM, Meijer JH (2003) Electrophysiology of the circadian pacemaker in mammals. Chronobiol Int 20:171-188.

Sehadová H, Sauman I, Sehnal F (2003) Immunocytochemical distribution of pigment-dispersing hormone in the cephalic ganglia of polyneopteran insects. Cell Tissue Res 312:113-125.

Shibata S, Oomura Y, Hattori K, Kita H (1984) Responses of suprachiasmatic nucleus neurons to optic nerve stimulation in rat hypothalamic slice preparations. Brain Res 302:83-89.

Shinohara K, Honma S, Katsuno Y, Abe H, Honma K (1995) Two distinct oscillators in the rat suprachiasmatic nucleus in vitro. Proc Natl Acad Sci USA 92:7396-7400.

Shinohara K, Hiruma H, Funabashi T, Kimura F (2000) GABAergic modulation of gap junction communication in slice cultures of the rat suprachiasmatic nucleus. Neuroscience 96:591-596.

Sokolove PG (1975) Localization of the cockroach optic lobe circadian pacemaker with microlesions. Brain Res 87:13-21.

Stengl M, Homberg U (1994) Pigment-dispersing hormone-immunoreactive neurons in the cockroach Leucophaea maderae share properties with circadian pacemaker neurons. J Comp Physiol [A] 175:203-213.

Strecker GJ, Wuarin JP, Dudek FE (1997) GABA $_{\mathrm{A}}$-mediated local synaptic pathways connect neurons in the rat suprachiasmatic nucleus. J Neurophysiol 87:2217-2220.

Tamada Y, Tanaka M, Munekawa K, Hayashi S, Okamura H, Kubo T, Hisa Y, Ibata Y (1998) Neuron-glia interaction in the suprachiasmatic nucleus: a double labeling light and electron microscopic immunocytochemical study in the rat. Brain Res Bull 45:181-187.

Tepper JM, Martin LP, Anderson DR (1995) GABA receptor-mediated inhibition of rat substantia nigra dopaminergic neurons by pars reticulata projection neurons. J Neurosci 15:3092-3103.

Van den Pol AN, Tsujimoto KL (1985) Neurotransmitters of the hypothalamic suprachiasmatic nucleus: immunocytochemical analysis of 25 neuronal antigens. Neuroscience 15:1049-1086.

Wagner S, Castel M, Gainer H, Yarom Y (1997) GABA in the mammalian suprachiasmatic nucleus and its role in diurnal rhythmicity. Nature 387:298-603.

Závodská R, Sauman I, Sehnal F (2003) Distribution of PER protein, pigmentdispersing hormone, prothoracicotropic hormone, and eclosion hormone in the cephalic nervous system of insects. J Biol Rhythms 18:106-122. 